\title{
Lessons from a respiratory illness outbreak in an aged-care facility
}

\author{
John Turahui ${ }^{\mathrm{A}}$, Cate Wallace ${ }^{\mathrm{B}}$, Paul Corben ${ }^{\mathrm{A}, \mathrm{C}}$ and Robin Osborne ${ }^{\mathrm{A}}$ \\ A Public Health Unit, North Coast Area Health Service \\ ${ }^{\mathrm{B}} \mathrm{NSW}$ Public Health Officer Training Program, NSW Department of Health \\ ${ }^{\mathrm{C}}$ Corresponding author.Email: paul.corben@ncahs.health.nsw.gov.au
}

\begin{abstract}
This report outlines practical lessons learnt from an influenza-like outbreak in an aged-care facility in NSW, which affected 26 residents, resulted in 14 hospital admissions and was associated with six deaths. No common causative agent was identified. Key recommendations include: encouraging agedcare facilities to establish mechanisms that improve the early identification of outbreaks and timely implementation of outbreak control strategies; identifying strategies to inform general practitioners of outbreaks if they have patients residing in aged-care facilities; and improving the vaccination coverage of the agedcare workforce.
\end{abstract}

\section{Outbreak in an aged-care facility}

Respiratory outbreaks in residential aged-care facilities are the cause of significant mortality and morbidity. ${ }^{1,2}$ An outbreak of an influenza-like illness occurred among residents and staff of a multi-purpose residential aged-care facility located on the north coast of New South Wales (NSW) in September-October 2006. At the time of the outbreak, there were 60 residents, ranging in age from 64 to 96 years, and 75 staff. The facility caters for residents with low- and high-care needs, and includes a purpose-built dementia facility. A high proportion of residents had previously been vaccinated against influenza A and B ( $96 \% ; n=58)$; however, vaccination coverage was low among staff members $(27 \% ; n=20)$.

\section{Public health response} Course of the outbreak

The outbreak extended over a 7-week period starting on 2 September 2006, with onset of the last case reported on 20 October 2006. A total of 26 residents (43\%) and one staff member $(1 \%)$ were affected. Of these, 14 residents $(54 \%)$ were hospitalised and six $(23 \%)$ died.

\section{Immediate actions taken by the public health unit} and aged-care facility

On 17 October 2006 the North Coast Area Health Service (NCAHS) Public Health Unit (PHU) was notified of the outbreak. The aged-care facility was advised to implement several strategies to contain the outbreak including: isolating sick residents; excluding sick staff; increasing infection control practices; deferring admissions; and discouraging non-essential visitors to the facility. The facility was also provided with fact sheets and the Guidelines for the prevention and control of influenza outbreaks in residential care facilities in Australia. ${ }^{3}$

During a site visit on the day following notification to the PHU, nasal and throat swabs and blood samples were taken from ill residents at the facility and from residents who had been admitted to hospital. In addition, PHU staff collected and reviewed information about affected staff and residents, including basic demographics, symptoms and onset date from those who had experienced symptoms since 2 September 2006. PHU staff also found that the facility was experiencing difficulty in sourcing protective (P2) masks. These are high efficiency, disposable masks with the capacity 
Table 1. Frequency of symptoms among affected residents and staff at a residential aged-care facility on the north coast of NSW, September-October $2006(N=27)$

\begin{tabular}{lc}
\hline Symptoms & Number affected \\
\hline Temperature $>38^{\circ} \mathrm{C}$ & 27 \\
Cough & 24 \\
Fatigue & 25 \\
Weakness & 18 \\
Aches and pains & 4 \\
Chills & 18 \\
Runny nose & 5 \\
Sore throat & 1 \\
Shortness of breath & 1 \\
Diarrhoea & 1 \\
Vomiting & 2 \\
\hline
\end{tabular}

to filter up to $95 \%$ of airborne particles with an aerodynamic diameter of 0.3 micron or more. The PHU was able to provide additional mask stocks.

Approximately 30 general practitioners who provided care to residents of the facility were notified of the outbreak by a letter forwarded by the facility. Antibiotics were prescribed for some symptomatic and asymptomatic residents by their general practitioners. Where possible, convalescent serology was collected 4 weeks later.

\section{Diagnosis and testing}

After considering relevant national guidelines, the range of symptoms reported and the occurrence of laboratoryconfirmed influenza in surrounding locales, a case definition was formed. ${ }^{3}$ For this outbreak, a case was defined as a resident or staff member of the facility who had a fever of $38^{\circ} \mathrm{C}$ or more, and at least two of the following symptoms with onset after 2 September 2006: chills, cough, fatigue, aches and pains (including severe pain), diarrhoea, vomiting and hypertension. A summary of the symptoms experienced by affected people is presented in Table 1 .

Symptomatic residents received point-of-care tests for influenza A and B during an initial site visit. Point-of-care tests, or tests that occur at the patient's beside, allow physicians to diagnose patients more rapidly than traditional laboratory-based testing. The Victorian Infectious Diseases Reference Laboratory tested nasal and throat swabs and blood samples taken from sick residents at a later date, for a range of conditions including: influenza A and B, parainfluenza, respiratory syncytial virus, adenovirus, Chlamydophila psittaci, Mycoplasma pneumoniae and Legionella pneumophila. Test selection was guided by avian and pandemic influenza protocols and Guidelines for the prevention and control of influenza outbreaks in residential care facilities, as well as potentially relevant environmental features of the facility, such as water fountains and domestic birds. ${ }^{3-5}$

\section{Ongoing response to the outbreak}

Two days after the outbreak notification, on 19 October, a teleconference was convened to discuss: the status of the outbreak, action taken, potential sources of infection and future management of the outbreak. The discussion group consisted of: the facility's Acting Director of Nursing; the Director of Medical Services and the infection control practitioner from the local hospital; and staff from the PHU and the Communicable Diseases Branch of NSW Department of Health. The possibility of further hospital admissions and the range of pathology tests to be undertaken were also discussed.

Throughout the outbreak, the PHU was in regular (at least daily) contact with the facility and the local hospital, and provided updates on the status of the outbreak, the likelihood of further hospitalisations and the health status of residents at the facility.

A second site visit was conducted on 20 October in order to:

- review the effectiveness of the infection control strategies implemented by the facility

- identify potential sources of infectious agents

- interview residents and staff members

- collect additional nasal and throat swabs and blood samples from ill and convalescing residents.

Samples were initially sent to the local pathology service for analysis. However, due to the number of sample batches, the range of tests required and the need for rapid processing, the samples were forwarded to the Victorian Infectious Diseases Reference Laboratory for analysis, as described above. Where possible, convalescent serology was collected 4 weeks after the first visit.

The results of the serological tests were inconclusive and no common causative agent was identified. One of six point-of-care tests performed was positive for both influenza A and B; however, the sensitivity and specificity of these tests is limited. ${ }^{6}$ Serology of convalescent patients also failed to identify a common causative agent.

\section{Media issues}

The outbreak received considerable media interest. Initially the aged-care facility was reluctant to make any public comment. Following increasing media speculation about the nature and extent of the outbreak, the NCAHS issued a statement on 30 October 2006 confirming that an outbreak of an unidentified infectious respiratory illness had been associated with the death of elderly people in residential care and requesting unwell people to avoid visiting agedcare facilities and health-care facilities. The media statement did not identify the aged-care facility nor provide details of the number of suspected cases or deaths. In response, a local newspaper used funeral notices from the previous week to speculate that up to 13 people over the age 
of 80 had died as a result of the outbreak. The following day the parent company of the aged-care facility issued a statement that appeared to conflict with the NCAHS statement regarding the link between recent resident deaths and the respiratory illness. This statement aroused further media interest and led to the involvement of the NSW Shadow Minister for Health. The local Member of Parliament wrote to the NSW Minister for Health requesting that the matter be investigated.

\section{Public health lessons}

Interaction between public health units and aged-care facilities

Several valuable lessons were learnt as a result of this outbreak. Most importantly, it highlights the need either to meet with aged-care providers regularly or involve them in periodic emergency management training sessions. This regular contact with aged-care facilities would emphasise the importance of early outbreak notification and close liaison with the PHU on matters such as vaccination and infection control. In this outbreak, the delay in the facility identifying and notifying the PHU of the outbreak may have contributed to difficulties in containing its spread and led to increased media interest. With support from PHUs, aged-care facilities should be encouraged to establish sentinel surveillance systems to improve outbreak recognition and timely implementation of infection control strategies. The NCAHS PHU has written to all residential aged-care facilities in the region to advise them of the resources available to assist in dealing with outbreaks, including notification procedures.

\section{Laboratory testing}

Considerable delay was experienced in receiving serological test results. Increased clarity about the tests available at reference laboratories and improved efficiency in transporting specimens to laboratories during an outbreak may shorten turnaround times for test results and aid the identification of appropriate treatment and infection control measures. The management of this outbreak would have benefited from closer liaison between the PHU and pathology laboratories, and the advocacy of the NSW Public Health Laboratory Liaison Officer when laboratory-related issues arose.

\section{Informing general practitioners}

Appropriate strategies for informing general practitioners who have patients residing in affected facilities need to be identified. In the case of this outbreak, the only available means of notifying the general practitioners of the residents of the outbreak was by a letter from the facility. This emphasises the need for a collaborative response to outbreaks between aged-care facilities, attending general practitioners and PHUs. It also reinforces the need for collaboration with Divisions of General Practice and aged-care panels and committees, where they exist, and the importance of seeking the consent of residents and the facility for information-sharing between agencies.

\section{Controlling the outbreak}

Although influenza was not identified as the likely causative agent in this outbreak, it provides important incidental lessons for influenza control in aged-care facilities. Researchers have reported that elderly residents of agedcare facilities often have an impaired immune response to influenza vaccinations due to age or co-morbidities. ${ }^{7,8}$ Influenza outbreaks have been reported in aged-care facilities with high levels of vaccination coverage (more than $85 \%$ ), even when the vaccine used matches the circulating influenza strain. ${ }^{9,10}$ In this facility, influenza vaccination coverage among residents was high (96\%); however, vaccination levels among staff were low (27\%). This low vaccination rate may in part be because influenza vaccinations are not provided free of charge to staff of aged-care facilities. One high priority for preventing influenza outbreaks in aged-care facilities is to prevent the introduction of the virus into the facility by ensuring high levels of vaccination coverage among all health-care workers and visiting general practitioners. To achieve this outcome, incentives for facilities to fund staff vaccinations could be considered. Increasing vaccination coverage among visitors is harder to achieve. One strategy is to educate the general public about the risks of transmitting respiratory illnesses when visiting health-care facilities. Appropriate signage at aged-care facilities and advice in the media during outbreak events are additional potential strategies that would reinforce these messages.

\section{Liaison between care and health organisations}

A standardised notification procedure to a defined point within the Australian Government Department of Health and Ageing would assist PHUs and the NSW Department of Health to provide timely outbreak notification. Identification of an appropriate mechanism to advise the NSW Ambulance Service of outbreaks in private facilities is also necessary.

Managing the outbreak through regular liaison with the affected facility, local hospital and PHU was considered beneficial for all, as this allowed joint problem-solving and information sharing. It also assisted in planning for potential hospital admissions and discharges. It would have been useful for this group to have jointly issued a media release to avoid the controversy that ensued following the perceived contradiction between the PHU and the facility's media releases.

\section{Summary}

Respiratory illness outbreaks in residential aged-care facilities cause significant mortality and morbidity. This outbreak resulted in 14 hospital admissions and was associated with six deaths among elderly residents. No common causative 
agent was identified. The practical lessons learnt that may be of use to other PHUs include:

- encouraging aged-care facilities to establish mechanisms for effective early identification of outbreaks and timely implementation of infectioncontrol strategies

- identifying effective strategies to inform general practitioners, the NSW Ambulance Service and the Australian Government Department of Health and Ageing of outbreaks in aged-care facilities

- improving the vaccination coverage among the agedcare health workforce.

\section{References}

1. Guy RJ, Di Natale R, Kelly H, Lambert S, Tobin S, Robinson P et al. Influenza outbreaks in aged-care facilities: staff vaccination and the emerging use of antiviral thereapy. Med J Aust 2004; 180: 640-2.

2. Ellis S, Coffey C, Mitchel E, Dittus R, Griffin M. Influenzaand respiratory syncytial virus-associated morbidity and mortality in the nursing home population. J Am Geriatr Soc 2003; 51: 761-7. doi:10.1046/j.1365-2389.2003.51254.x

3. Communicable Disease Network Australia. Guidelines for the prevention and control of influenza outbreaks in residential care facilities in Australia. Canberra: Department of Health and Ageing; 2005.
4. NSW Health. NSW Health Pandemic Influenza Interim Response Protocol for Public Health Units 2006. Sydney: NSW Health; 2006. Available from: http://www.health.nsw.gov.au/ pandemic/docs/piresponse.pdf (Cited 19 March 2007.)

5. Department of Health and Ageing. Australian Management Plan for Pandemic Influenza, June 2005. Canberra: Department of Health and Ageing; 2005. Available from: http://www.health.gov.au/internet/main/publishing.nsf/ Content/FC517607D6EE443ECA2570190019CDF7/\$File/ pandemic_plan.pdf (Cited 24 October 2008.)

6. Charles PG, Grayson ML. Point of care tests for lower respiratory tract infections. Med J Aust 2007; 187(1): 36-9.

7. National Health and Medical Research Council. The Australian immunisation handbook. 8th ed. Canberra: NHMRC; 2003. Available from: http://www9.health.gov.au/immhandbook/ pdf/handbook.pdf (Cited January 2007.)

8. Bradley SF. Prevention of influenza in long-term-care facilities. Long-Term-Care Committee of the Society for Healthcare Epidemiology of America. Infect Control Hosp Epidemiol 1999; 20: 629-37. doi:10.1086/501687

9. Morens DM, Rash VM. Lessons from a nursing home outbreak of influenza A. Infect Control Hosp Epidemiol 1995; 16: 275-80.

10. Drinka PJ, Gravenstein S, Krause P, Schilling M, Miller BA, Shult P. Outbreaks of influenza A and B in a highly immunized nursing home population. J Fam Pract 1997; 45: 509-14. 